\title{
Standardization of Artificial Intelligence for the Development and Use of Intelligent Systems
}

\author{
Evgeniy Bryndin Russia \\ Research Department, Research Center "Natural Informatics", Novosibirsk, Russia
}

Email address:

bryndin15@yandex.ru

\section{To cite this article:}

Evgeniy Bryndin Russia. Standardization of Artificial Intelligence for the Development and Use of Intelligent Systems. Advances in Wireless Communications and Networks. Vol. 6, No. 1, 2020, pp. 1-9. doi: 10.11648/j.awcn.20200601.11

Received: July 29, 2020; Accepted: August 12, 2020; Published: August 27, 2020

\begin{abstract}
Standard is document that establishes the requirements, specifications, guidelines or characteristics according to which materials, products, processes and services that are suitable for these purposes can be used. ISO has more than 21,000 international standards covering aspects of technology and business. Standardization of artificial intelligence is advisable to start with concepts. The standard of artificial intelligence can be defined as follows. Artificial intelligence is a scientific applied direction for the development and creation of technological and software cognitive complexes of the digital twin of human intelligence, capable of learning, retraining, self-realization and development on the basis of the criterion of preferences and to improve functional activities by quality choice and mastering creative innovative high-tech artificial intelligence, a roadmap is being developed. The main tasks of standardization of artificial intelligence are, firstly, the unification and standardization of terminology, ensuring the interoperability of artificial intelligence systems, ensuring methodological continuity in the field of artificial intelligence methods and algorithms, and improving the effectiveness of collective work on creating artificial intelligence systems. Secondly, the removal of regulatory barriers related to the processing of personal data. Thirdly, standardization of procedures for confirming the predictability of the behavior of the artificial intelligence system under certain operating conditions, standardization of procedures for confirming the safety of the functioning of the artificial intelligence system. Fourth, unification of quality characteristics of artificial intelligence systems aimed at solving specific application problems. Fifth, standardization of requirements for technology platforms with artificial intelligence, development of standard artificial intelligence cases for the development and use of intelligent systems and technologies. Artificial intelligence technologies are already ripe enough to standardize them. Without it, the industry will not be able to develop normally, the interests of the state and society will not be protected. The United States, China, other countries and international organizations have begun active work in this direction. Standardization of artificial intelligence will help to find boundaries in which artificial intelligence will benefit humanity and not harm.
\end{abstract}

Keywords: Standardization, Strong Artificial Intelligence, Security of Intelligent Systems, Ethical Aspects

\section{Introduction}

Standards as the main technical basis of economic and social activities have already become an important indicator for measuring the level of technological development in a country or region, are the main benchmarks for products entering the market, and are a concrete manifestation of the market competitiveness of enterprises. Standardization plays fundamental, supportive and leading role in artificial intelligence and its industrial development [1]. This is both a key starting point for promoting industrial innovation and development and a key point in industrial competition. The promotion and improvement of artificial intelligence standards is associated with the development of the industry and the competitiveness of products in the international market.

Developed countries such as the United States, the European Union and Japan attach great importance to the standardization of artificial intelligence. National Strategic Plan for Artificial Intelligence Research and Development, released by the United States; Human Brain Project, released by the European Union, and (AIP Project), implemented by Japan, put forward a number of proposals that focus on basic technologies, advanced talent, standard specifications and 
other ways to strengthen the deployment of artificial intelligence.

China attaches great importance to standardization of artificial intelligence. The plan for the development of new generation artificial intelligence (AIDP) of the State Council proposed the principles of safety, accessibility, compatibility and control. The Ministry of Industry and Information Technology indicated that it is necessary to create a system of specification of industry standards for artificial intelligence and to establish and improve such technical standards as general fundamentals, compatibility, security and confidentiality, as well as industrial applications, and at the same time build systems for evaluating artificial intelligence products.

In general, more attention should be paid to the important leading role that standardization of artificial intelligence plays in promoting technological innovation and supporting industrial development. Standardization work helps accelerate the introduction of artificial intelligence technologies and the commercialization of research results. At this stage, artificial intelligence technology is developing rapidly [2-26]. As products and applications emerge on the market that can be progressively expanded, technological advances should use standardized methods to specify technological outcomes and quickly advance innovation. Standardization work helps improve the quality of artificial intelligence products and services. Whether it is for facial recognition systems, smart speakers, service robots or other products that appear on the market, if the quality of the products is poor, they need standard unified specifications, and the products and quality of services will be improved in accordance with the methods of testing and conformity assessment.

Standardization can help to effectively protect the security of users, protect the rights and interests of users. This requires that intellectual systems conform to and serve human ethics and provide information security.

Standardization will help create a fair and open environment in the field of artificial intelligence. This requires a unified standard to ensure compatibility and coordination among manufacturers to prevent monopolies in industry, block users and create a profitable industrial environment.

The first, second and third sections of the article are devoted to aspects of the safety of artificial intelligence. The fourth section discusses the ethical aspects of artificial intelligence. The fifth section covers the protection of privacy by artificial intelligence. The sixth section describes aspects of an interdisciplinary approach to artificial intelligence. Modern concepts and terminology of artificial intelligence are discussed in the seventh section of the article.

\section{Security Aspects of Systems with Artificial Intelligence}

For road safety, traffic rules apply, in medicine there is a
Hippocratic rule (do not harm), in production there are safety rules. The practical use of artificial intelligence systems in various areas of society also requires the introduction of safety rules and standardization. Safety in the field of artificial intelligence is related to ensuring that an artificial intelligence-based system is tested in conditions close to the real world, so that its goals and behavior are properly adjusted, and the system can be safely implemented in society. The discrepancy between the developer's goal and how the intelligent agent interprets it can cause unexpected consequences that become apparent only when the system begins to be applied in the real world. The competition to bring systems based on artificial intelligence to the market encourages developers to verify and confirm the parameters of experimental software, without which the security of deploying intelligent agents cannot be guaranteed. For example, a poorly engineered portfolio management intellectual agent whose goal is to maximize profits by investing in assets with a specific investment risk category can operate according to test settings in a project environment where the agent is offered several assets for selection, pre-selected by developers. However, after launching in the real world, the agent can behave unexpectedly, finding that investment income can be maximized, primarily by investing available capital resources in illegal activities.

As the number of systems connected to the Internet grows, cyber security will have an increasing economic impact on society. Already, they are estimated at tens of billions of dollars, so the advent of increasingly advanced artificial intelligence systems, on which society will depend, will increase the negative impact of those that do not guarantee security, even more. However, recently, security in the field of artificial intelligence has gained recognition and has begun to gain momentum, especially in the scientific community, showing cumulative annual growth in investment.

Artificial intelligence has plenty of ways to help solve problems and strengthen the security of citizens. Artificial intelligence can increase the productivity and efficiency of public safety agencies through automation. Artificial intelligence is able to identify people, objects and movements, to send alarms when identifying threats or anomalies in data. This is important in a variety of cases, including ensuring the safety of major public events, patrolling roads and monitoring centers of criminal activity. Most information security analytics and solutions are based on machine learning. The main principle of its work is that systems are trained on large sets of specially selected data and in the future they apply the acquired knowledge. The system evaluates the operation parameters, compares them with existing profiles, detects suspicious signs and issues recommendations.

\section{Approach to Standardization of Safe Use of Artificial Intelligence}

The safety of systems with artificial intelligence and 
cognitive robots is relevant for smart factories, health centers, cafes, services, vehicles, agro-industrial complex, defense industry, etc. [1-26].

Modern artificial intelligence methods (neural networks, machine learning) and science cannot explain how a trained system makes a decision. Because of this, the price of error in the areas: transport, medicine, defense, etc. Artificial intelligence specialists configure artificial neural networks using coefficients in the process of their training with formalized data to obtain the necessary result. For the safe use of an artificial neural network, it is necessary to determine the range of changes in the attributes of the input data.

In order to apply artificial intelligence safely everywhere, it is necessary to standardize the use of artificial intelligence.

Consider briefly the approach to national standardization of the safe use of artificial intelligence by category: classification 1.11.022-1.021.19 "GOST R. Intelligent data processing technologies" and by case: A. 111 Application of Strong Artificial Intelligence-"ISO/IEC JTC 1/SC 42/WG 4 NO 254 TR 24030 working draft v10"-iso

Must enter the term and definition intellectual ensemble: intelligent ensemble-complex of compatible intelligent systems interacting through an intelligent interface, implementing either a technological process, social services, multidisciplinary interdisciplinary research, or a production cycle.

It is necessary to add types of classification diversification and mobility in 1.11.022-1.021.19 "GOST R. Intelligent data processing technologies, Classification", Section 5.3 Association of Classification Types with AI Life Cycle Stages:

diversification-expanding the functions of artificial intelligence and mastering a new type of functionality in order to increase the efficiency, quality and functional diversity of the intellectual system; mobility-the ability of the ensemble of intellectual agents to quickly functional retraining and the development of its intelligence.

Definitions diversification and mobility align with life cycle definitions and life cycle model.

Life cycle-development of a system, products, service, project or other human-made entity from design to write-off.

Life cycle model-the structural basis of life cycle processes and actions, which also serves as a common reference for linking and understanding.

Images and numbers are used as simulation data. The environment is perceived through images and scenes. Scenes consist of a number of images. Scenes are static (paintings) and dynamic. Dynamic scenes are characterized by patterns of behavior of objects and objects. The patterns are either described by formulas or presented in a graph (numerical way). The safety of behavior depends on spatial, temporal, subject, visual and sound sensitivity. Behavior security is ensured within the boundaries of image similarity in a safe environment.
Modeling shows that the development of behavioral skills (mobility) and professional skills (diversification) increases the sensitivity of environmental perception, reduces risks, and increases safety.

Define the basic concepts:

artificial intelligence-the ability of the system to acquire, process, apply and diversify knowledge based on previous experience in solving specific problems related to the processing of data attributes and the mobility of an intelligent system.

Attributes of data-objects, objects, materials, things, processes, the phenomena and other aspects of the physical world have various properties and characteristics.

Properties are represented by qualitative attributes. Characteristics appear to be meaningful attributes. The qualitative attribute can be visual or sound. The meaningful attribute may be represented by a number, a language sense, a visual or sound image, a mathematical or behavioral action, or an algorithm. Meaningful qualitative attributes are big clever data of artificial intelligence.

Big Smart Data (Big SD) is collection of qualitative and quantitative attributes associated in time, space, and subject area.

The attributes of smart data (numbers and images) form an idea of the world. Big SD attributes of the fields of economics, industrial industries, technologies and professions help to build and train artificial neural multilayer artificial intelligence networks for managing, making decisions and making recommendations to specialists and managers. Applied research modeling helps to accumulate Big SD scientific attributes in real time and simultaneously use them to deeply train multilayer artificial neural networks of intelligent modeling management agents, make decisions, and make recommendations to researchers. By modeling, the optimal (equilibrium safe) state of artificial neural networks of intelligent agents and the limits of attribute values relative to this state are determined. Numeric attribute values define numeric limits. For visual and sound images, limits of similarity to the optimal image are revealed.

Safety standards of artificial intelligence should also ensure reliable control of the conservation of nature and the ecology of living spaces by used industrial technologies.

\section{Standard Case Application of Strong Artificial Intelligence}

Application technologies work using strong artificial intelligence [21-26]. Case for the use of strong artificial intelligence approved by the Japanese Technical Committee for the Standardization of Artificial Intelligence and is the international standard A. 111 Application of Strong Artificial Intelligence-"ISO/IEC JTC 1/SC 42/WG 4 NO 254 TR 24030 working draft v10"-ISO/IEC 24030:2019 (E). See the case on the use of strong artificial intelligence below. 
Table 1. General.

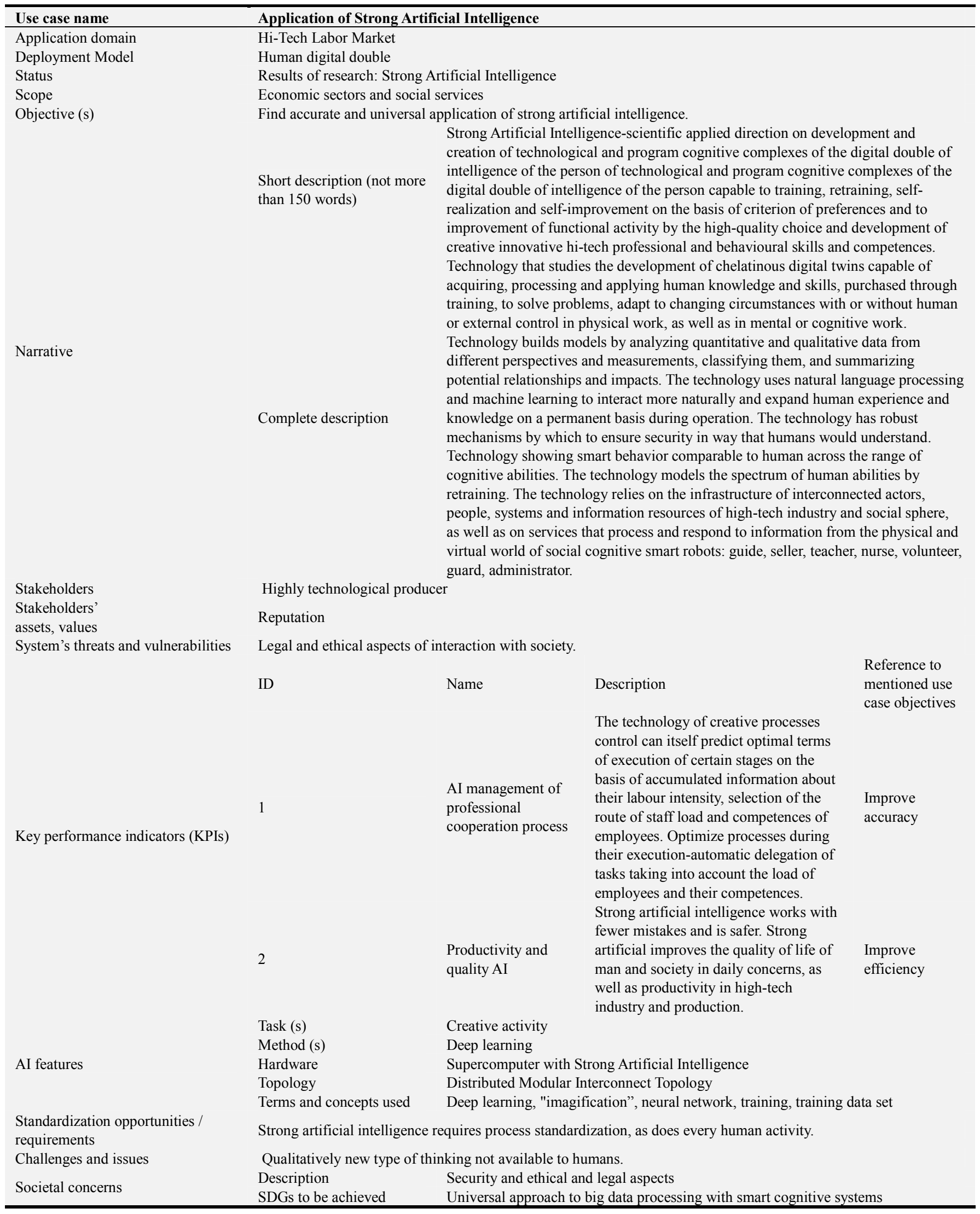


Table 2. Data.

\begin{tabular}{ll}
\hline Data characteristics & \\
\hline Description & Strong Artificial Intelligence Data \\
Source & Model and technology of Strong Artificial Intelligence \\
Type & Strong \\
Volume (size) & Hi-Tech Labor Market \\
Velocity (e.g. real time) & Supercomputering Velocity \\
Variety (multiple datasets) & streams of multiple datasets \\
Variability (rate of change) & Retraining \\
Quality & High \\
\hline
\end{tabular}

Table 3. Process scenario.

\begin{tabular}{|c|c|c|c|c|c|}
\hline \multicolumn{6}{|c|}{ Scenario conditions } \\
\hline N. & Scenario name & Scenario description & Triggering event & Pre-condition & Post-condition \\
\hline 1 & Training & $\begin{array}{l}\text { Train a model (deep neural } \\
\text { network) with training data set }\end{array}$ & $\begin{array}{l}\text { Technological process raw } \\
\text { data set is ready }\end{array}$ & Formatting of data & Management of safety \\
\hline 2 & Evaluation & Expansion of the trained model & $\begin{array}{l}\text { Development of } \\
\text { technological thinking and } \\
\text { behaviour }\end{array}$ & $\begin{array}{l}\text { Cognitive thinking patterns } \\
\text { and psychological behaviors }\end{array}$ & $\begin{array}{l}\text { Meeting KPI requirements is } \\
\text { condition of development }\end{array}$ \\
\hline 3 & Execution & Model and Technology Tooling & Interaction & Activization of Model & Completion of interaction \\
\hline 4 & Retraining & $\begin{array}{l}\text { Retrain a model with training } \\
\text { data set }\end{array}$ & $\begin{array}{l}\text { Certain period of time has } \\
\text { passed since the last } \\
\text { training/ retraining }\end{array}$ & $\begin{array}{l}\text { Additional data and } \\
\text { knowledge }\end{array}$ & $\begin{array}{l}\text { Combining Data and } \\
\text { Knowledge }\end{array}$ \\
\hline
\end{tabular}

Table 4. Training.

\begin{tabular}{|c|c|c|c|c|c|}
\hline Scenario name & Training & & & & \\
\hline Step No. & Event & $\begin{array}{l}\text { Name of } \\
\text { process/Activity }\end{array}$ & Primary actor & Description of process/activity & Requirement \\
\hline 1 & $\begin{array}{l}\text { Sample raw data set is } \\
\text { ready }\end{array}$ & $\begin{array}{l}\text { Specification and } \\
\text { classification }\end{array}$ & Manufacturer & Transform sample raw data & $\begin{array}{l}\text { Strong AI } \\
\text { Software }\end{array}$ \\
\hline 2 & Completion of Step 1 & $\begin{array}{l}\text { Creating Set of } \\
\text { Experimental Data }\end{array}$ & Manufacturer & $\begin{array}{l}\text { Development of set of } \\
\text { experimental data through job } \\
\text { modelling }\end{array}$ & $\begin{array}{l}\text { Software of } \\
\text { modelling }\end{array}$ \\
\hline 3 & Completion of Step 2 & Model training & AI solution provider & $\begin{array}{l}\text { Train a model (deep neural } \\
\text { network) with experimental data } \\
\text { set created by Step } 2\end{array}$ & \\
\hline
\end{tabular}

Table 5. Evaluation

\begin{tabular}{|c|c|c|c|c|c|}
\hline Scenario name & Evaluation & & & & \\
\hline Step No. & Event & $\begin{array}{l}\text { Name of } \\
\text { process/Activity }\end{array}$ & Primary actor & Description of process/activity & Requirement \\
\hline 1 & $\begin{array}{l}\text { Completion of } \\
\text { training/retraining }\end{array}$ & Research & Manufacturer & $\begin{array}{l}\text { Train model (deep neural network) with } \\
\text { experimental data set created }\end{array}$ & \\
\hline 2 & Completion of Step 1 & Identification & AI solution provider & $\begin{array}{l}\text { Based on data, detect execution using a deep } \\
\text { neural network trained in learning scenario }\end{array}$ & \\
\hline 3 & Completion of Step 2 & Evaluation & Manufacturer & $\begin{array}{l}\text { Comparison of phase } 2 \text { results with human } \\
\text { performance }\end{array}$ & \\
\hline \multicolumn{6}{|c|}{ Input of evaluation } \\
\hline
\end{tabular}

Table 6. Execution

\begin{tabular}{llllll}
\hline Scenario name & Execution & Name of \\
Step No. & Event & $\begin{array}{l}\text { Primary } \\
\text { actor }\end{array}$ & Description of process/activity & Requirement \\
\hline 1 & $\begin{array}{l}\text { Completion of comparison of } \\
\text { modeling results with human } \\
\text { performance }\end{array}$ & Research & Manufacturer & $\begin{array}{l}\text { Development of a set of } \\
\text { experimental data through job } \\
\text { modelling }\end{array}$ & $\begin{array}{l}\text { Based on modified data train } \\
\text { model (deep neural network) with } \\
\text { experimental data set created } \begin{array}{l}\text { The trained model with } \\
\text { do be handed over to the } \\
\text { manufacturer }\end{array}\end{array}$ \\
\hline $\begin{array}{l}\text { Input of Execution } \\
\text { Output of Execution }\end{array}$ & Completion of Step 1 & Identification & Manufacturer & \\
\hline
\end{tabular}


Table 7. Retraining.

\begin{tabular}{|c|c|c|c|c|c|}
\hline \multirow{2}{*}{$\begin{array}{l}\text { Scenario name } \\
\text { Step No. }\end{array}$} & \multicolumn{5}{|l|}{ Retraining } \\
\hline & Event & $\begin{array}{l}\text { Name of process/ } \\
\text { Activity }\end{array}$ & $\begin{array}{l}\text { Primary } \\
\text { actor }\end{array}$ & Description of process/activity & Requirement \\
\hline 1 & $\begin{array}{l}\text { Certain period of time has passed } \\
\text { since the last training/retraining }\end{array}$ & Research & Manufacturer & Additional data and knowledge & \\
\hline 2 & Completion of Step 1 & $\begin{array}{l}\text { Experimental data set } \\
\text { creation }\end{array}$ & Manufacturer & $\begin{array}{l}\text { Combining Data and Knowledge } \\
\text { Based on modified data train model } \\
\text { (deep neural network) with } \\
\text { experimental data set created }\end{array}$ & \\
\hline 3 & Completion of Step 2 & Model training & $\begin{array}{l}\text { AI solution } \\
\text { provider }\end{array}$ & $\begin{array}{l}\text { Comparison of phase } 2 \text { results with } \\
\text { human performance }\end{array}$ & \\
\hline \multicolumn{2}{|c|}{ Specification of retraining data } & \multicolumn{4}{|c|}{ Retraining data set has to include recent data } \\
\hline
\end{tabular}

\section{Ethical Aspects of Artificial Intelligence}

Artificial intelligence is a continuation of human intelligence, as well as a continuation of the system of human values. In its development, it should include a proper consideration of human ethical values. The establishment of ethical requirements for artificial intelligence technology is based on public consensus on the ethics of artificial intelligence. First, the ultimate goal of artificial intelligence should be the good of man. Secondly, a clear system of responsibility should be established, both with regard to the development and application of artificial intelligence technologies. Technology development should be based on standards, and the application of technology should be based on responsibility.

Thirdly, the principle of consistency of rights and obligations. It is necessary to properly record the necessary commercial data, monitor the appropriate algorithm and conduct a reasonable review of commercial applications; On the other hand, businesses can still use reasonable intellectual property rights or trade secrets to protect the basic parameters of an enterprise. In the field of artificial intelligence, the principle of "consistency of rights and obligations" has not yet been fully implemented by the business community and the government in ethics practice. This is mainly due to the lack of ethical standards for artificial intelligence, so engineers and design teams do not take into account ethical aspects when developing and producing artificial intelligence products and services. In addition, the entire artificial intelligence industry is not yet accustomed to a flow of operations that takes into account the needs of various stakeholders. In industries related to artificial intelligence, the protection of trade secrets is not balanced with transparency.

\section{Aspects of Artificial Intelligence Privacy}

A clear and effective definition of privacy is needed. The development of artificial intelligence methods also facilitates the violation of personal life, so relevant laws and standards should provide more powerful means of protecting personal life. Using artificial intelligence technology, it is easy to infer those aspects of confidentiality that citizens do not want to disclose, such as obtaining private information from public data and obtaining information from one person about other people (such as friends, relatives and colleagues).

In addition, the development of artificial intelligence technology makes it more convenient for the government to collect and use personal data of citizens. Large volumes of personal data can help government agencies better understand the status of the people they serve and guarantee the capabilities and quality of personalized service. However, it follows that sufficient attention should be paid to the risks and potential harm to the improper use of personal data by government departments and government employees.

It is necessary to revise the definition of acquisition and informed consent to receive personal data in the context of artificial intelligence. Above all, relevant policies, laws and standards should directly regulate the collection and use of data. Simply obtaining the consent of the data owner is not enough. Secondly, in order to protect the confidentiality of data sources, developers and developers should be provided with standard procedures that are practical and feasible and can be adapted to different conditions of use. Thirdly, we must begin to regulate the use of artificial intelligence, which could be used to obtain information that exceeds what citizens initially agreed to divulge. Finally, policies, laws, and standards should expand the protection of personal data management to encourage the development of appropriate technologies and to explore the use of algorithm tools as individuals in the digital and real world. This approach allows for both control and use, since the algorithm can set different usage permissions depending on different situations.

Technology development creates risks for public trust. How to increase social confidence and allow technology to follow ethical requirements, especially, this is an urgent problem that must be solved so that confidentiality is not violated. This requires the development of sound policies, laws and standards, as well as cooperation within the international community. Such policies should also contribute to a better understanding of artificial intelligence products and pay particular attention to the great benefits that this new technology brings to society, as well as to big problems. 


\section{Aspects of Interdisciplinary Approach to Standardization of Artificial Intelligence}

DIN and DKE provide the world's leading standardization platform. They organize standardization topics and coordinate collective work that goes beyond the activities of their own standard-setting organizations. DIN, a national standardization organization supported by the German industry, is actively working on the early development of open standards that establish an interdisciplinary approach to artificial intelligence. They will ensure widespread interaction between different disciplines that work with artificial intelligence, and specify the requirements for working with artificial intelligence. Identify specific framework conditions that are conducive to innovation and extend the applicability of artificial intelligence to industry and society at large while ensuring planning and legal certainty. As stated in the artificial intelligence strategy, standardization helps maintain the adequacy and flexibility of the regulatory framework.

Standardization at the R \& D stage reduces costs, increases investment security, and gives innovative solution providers information leadership over future competitors, helping them position their products more effectively in markets; For example, by establishing links with relevant actors in the standard-setting process. Standardization thus catalyses innovation by increasing market access and market penetration, as well as by internationalizing new technological advances.

The results of standardization activities are published in the public domain, which contributes to the dissemination of information and wide application. In addition, standardization helps prevent market fragmentation that hinders growth and avoid mutually agreed solutions. Standardization should be included in the financing of artificial intelligence technologies, both at the federal and regional levels, in order to strengthen these economically positive effects.

Standardization is a catalyst for innovation and should be integrated into all artificial intelligence financing programs. Whoever sets the standard controls the market. Researchers, businesses and policymakers should come together to ensure coordination of their activities in the field of artificial intelligence standards by responsible standardization organizations.

All artificial intelligence applications require a standardized approach that is widely accepted. It is the power of possible and endless interdisciplinary diversity that makes standardization of artificial intelligence mandatory. Only standards can define often very complex processes in a way that is beneficial to all producers and all sectors. Thus, standards provide the compatibility required for artificial intelligence technologies in applications ranging from selfdriving cars to smart cities and industry 4.0. Market-oriented standards and specifications ensure quality and ecology, as well as safety and communication.

\section{Modern Concepts and Terminology of Artificial Intelligence}

Artificial intelligence (AI) is scientific applied direction for the development and creation of technological and software cognitive complexes of the digital twin of human intelligence, capable of learning, retraining, self-realization and development based on the criterion of preferences and to improve functional activities by quality choice and mastering creative innovative high-tech professional and behavioral skills and competencies.

Big Data-data collected automatically is too large to be collected and processed manually, allowing you to find new patterns and knowledge that cannot be obtained from local data fragments.

Recommendation Systems-systems that provide recommendations based on implicit laws detected by AI algorithms using big data analysis.

Prognostic systems are systems that capture the relationships between variables in historical datasets and their totals.

Biometrics-taking and digitizing various biological characteristics of a person-faces, gestures, gait, fingerprints, blood pressure, temperature, etc.

Knowledge Management-represent knowledge of the world in the form of a hierarchy of concepts, such that the system can use knowledge to solve complex problems, such as diagnosing diseases, making decisions or talking to a person.

Man-Machine Interface-dialogue between a person and devices.

Virtual interlocutors-programs that communicate with a person in a natural language.

Machine Learning (ML) is AI area that creates algorithms that independently learn and predict the situation based on available data, without requiring programmer intervention.

Supervised Learning is type of machine learning where the learning algorithm is given pre-classified and sorted data, consisting of examples of input data and desired processing results

Unsupervised Learning-type of machine learning in which the learning algorithm does not include any classifications and labels, itself determines the structure and relationships of the input data.

Reinforcement Learning is type of machine learning in which the algorithm has a specific goal.

Deep Neural Networks is multilayer neural network in which "learned" data is passed through several layers of "neurons"; on each layer, the result of the previous layer is used as input information.

Convolutional Neural Networks-multilayer neural networks with an alternation of special convolutional (summing) and ordinary layers, the most effective for recognizing images.

Feed-Forward Networks is neural network with many layers where data spreads only forward. 
Recurved neural networks are deep neural networks where data can spread between layers forward and backward.

Training Set is set of precedents (objects, cases, events, subjects, texts, photographs, samples, etc.) chosen to teach algorithm from set of all possible precedents called general set.

Mark-up-Process-training selection to assign properties to objects in it that the algorithm learns to recognize.

Markup system is automated workplace that allows many machine learning operators to quickly mark out training samples.

Open data-archives, data libraries, usually stored on cloud storage and available for download anywhere in the world to anyone.

Data Processing Center-special technical room, which houses special equipment (servers, server racks, network equipment, etc.) for processing, storage and transmission of large amounts of data, with power supply and powerful communication channels.

Dataset is set of data having the same structure and combined in meaning.

Tagged data-containing structured information for all dataset instances, which include meta-information for solving specific applications.

Julia is high-level general-purpose programming language focused on high-performance numerical analysis and computational science.

Python is widely used programming language and can be used to implement artificial intelligence due to the simple and seamless structure that it offers.

Java-extremely popular cross-platform programming language, provides search algorithms and neural networks.

Tensorflow-open software library for machine learning developed by Google to solve the problems of building and training a neural network in order to automatically find and classify images, achieving the quality of human perception.

PyTorch is open source Python machine learning library based on Torch.

Keras-open neural network library written in Python.

Caffe is foundation of deep learning, created taking into account speed and modularity.

CUDA is parallel software and hardware architecture that dramatically increases computing performance with Nvidia GPUs.

Clouds-computing services (servers, storage, network, software) available over the Internet.

\section{Conclusion}

The coming years will take to increase safety and standardize the development and application of viable strong artificial intelligence. International standardization of the production and use of intelligent systems ensuring their compatibility has intensified. Intelligent systems can be combined at the software level through a standard module for communicating with them. Compatibility will allow you to produce from them intelligent ensembles with the cognitive and behavioral abilities of a person for service services. It will also allow the production of intelligent production hightech complexes. Standardization of a viable strong artificial intelligence will help ensure the interface, compatibility and synergy of its safe application in various sectors of the economy, industry and service. The development of approaches to the creation of artificial intelligence technologies that will be favorable for humanity remains one of the most pressing tasks. As criterion for the work of companies, the development and use of equilibrium safe intelligent systems should be applied to the field of artificial intelligence [19]. In the future, a reliable and safe creative viable strong artificial intelligence will significantly change the life of the world community.

\section{References}

[1] ISO/IEC Directives, Part 2, Rules for the structure and drafting of International Standards, 2004

[2] B. Du Boulay. "Artificial Intelligence as an Effective Classroom Assistant". IEEE Intelligent Systems, V 31, p. 7681. 2016.

[3] M. R. Sumner, B. J. Newendorp and R. M. Orr. "Structured dictation using intelligent automated assistants". N US $9,865,280,2018$.

[4] S. Hong. "Artificial intelligence audio apparatus and operation method thereof". N US 9,948,764, Available at: https://patents.google.com/patent/US20150120618A1/en. 2018.

[5] Evgeniy Bryndin. Digital technologies of the industry 4.0. Chepter 10, C. 201-222, Book: Computer Science Advances: Research and Applications. Nova Science Publisher. 2019. $252 \mathrm{c}$.

[6] Evgeniy Bryndin. Cognitive Robots with Imitative Thinking for Digital Libraries, Banks, Universities and Smart Factories. International Journal of Management and Fuzzy Systems. V. 3, N. 5, 2017, pp 57-66.

[7] Evgeniy Bryndin. Collaboration of Intelligent Interoperable Agents via Smart Interface. International Journal on Data Science and Technology, Vol. 5, № 4. 2019. Pages: 66-72.

[8] Evgeniy Bryndin. Social Cognitive Smart Robots: Guide, Seller, Lecturer, Vacuum Cleaner, Nurse, Volunteer, Security Guard, Administrator. Communications. V. 7, Issue 1. 2019. P. 6-12.

[9] Evgeniy Bryndin. Human Digital Doubles with Technological Cognitive Thinking and Adaptive Behaviour. Software Engineering, Volume 7, Issue 1, 2019. P. 1-9.

[10] Frank Chen, "AI, Deep Learning, and Machine Learning: A Primer," Andreessen Horowitz, June 10, 2016 , http://a16z.com/2016/06/10/ai-deep-learning-machines.

[11] Evgeniy Bryndin. Robots for Communication in Public in High-Tech Industry Life and Space. Frontiers Journal of Current Engineering Research. Vol. 1, Issue 1, 2019. P. 1-10.

[12] Evgeniy Bryndin. Mainstreaming technological development of industrial production based on artificial intelligence. COJ Technical \& Scientific Research, 2 (3). 2019. P. 1-5. 
[13] Evgeniy Bryndin. Robots with Artificial Intelligence and Spectroscopic Sight in Hi-Tech Labor Market. International Journal of Systems Science and Applied Mathematic, Vol. 4, № 3, 2019. Pages: 31-37.

[14] Evgeniy Bryndin. Increased Sensitivity and Safety of Cognitive Robot by Development of Professional and Behavioral Skills. International Journal of Research in Engineering. Volume-2, Issue-1, 2020. Pages: 14-21.

[15] Evgeniy Bryndin. Collaboration Robots with Artificial Intelligence as Digital Doubles of Person for Communication in Public Life and Space. Budapest International Research in Exact Sciences (BirEx-Journal), Volume 1, No. 4, 2019. P: 111.

[16] Evgeniy Bryndin Formation Smart Data Science for Automated Analytics of Modeling of Scientific Experiments. American Journal of Software Engineering and Applications. Vol. 8, Issue 2, 2019. P. 36-43.

[17] AI white paper editing committee of Information Technology Promotion Agency, Japan. "Artificial Intelligence White Paper" (in Japanese). Kadokawa Ascii Research Laboratories, Inc, 2017.

[18] Evgeniy Bryndin. Development of Artificial Intelligence by Ensembles of Virtual Agents on Technological Platforms. COJ Technical \& Scientific Research. 2 (4). 2020. Pages: 1-8.

[19] Evgeniy Bryndin. Practical Development of Creative LifeSaving Strong Artificial Intelligence. Communications, Volume. 7, Issue 2, 2019. Pages: 31-39.

[20] Preparing for the Future of Artificial Intelligence, Executive Office of the President, National Science and Technology Council Committee on Technology, October 2016.
[21] Evgeniy Bryndin. Transformation Modeling of Nanostructures by Cognitive Systems according to Big Smart Data. Chepter 3, P. 21-28. Book "New Materials: Preparation, Properties and Applications in the Aspect of Nanotechnology". USA: Nova Science Publisher. 2020. 250 p.

[22] Peter Stone, Rodney Brooks, Erik Brynjolfsson, Ryan Calo, Oren Etzioni, Greg Hager, Julia Hirschberg, Shivaram Kalyanakrishnan, Ece Kamar, Sarit Kraus, Kevin LeytonBrown, David Parkes, William Press, AnnaLee Saxenian, Julie Shah, Milind Tambe, and Astro Teller. "Artificial Intelligence and Life in 2030." One Hundred Year Study on Artificial Intelligence: Report of the 2015-2016 Study Panel, Stanford University, Stanford, CA, September 2016. Doc: http://ai100.stanford.edu/2016-report.

[23] Evgeniy Bryndin. Formation and Management of Industry 5.0 by Systems with Artificial Intelligence and Technological Singularity. American Journal of Mechanical and Industrial Engineering. Volume 5, Issue 2. 2020. pp. 24-30.

[24] Evgeniy Bryndin. Increase of Safety Use Robots in Industry 4.0 by Developing Sensitivity and Professional Behavioral Skills. American Journal of Mechanical and Industrial Engineering. Volume 5, Issue 1, 2020. Pages: 6-14.

[25] Evgeniy Bryndin. Formation of Technological Cognitive Reason with Artificial Intelligence in Virtual Space. Britain International of Exact Sciences Journal, 2 No. 2, 2020. P. 450461.

[26] ISO/IEC JTC 1/SC 42/WG 4 Use cases and applications Convenorship: JISC (Japan). 2019-12-23. https://isotc.iso.org/livelink/livelink/open/jtc1sc42 wg4. 